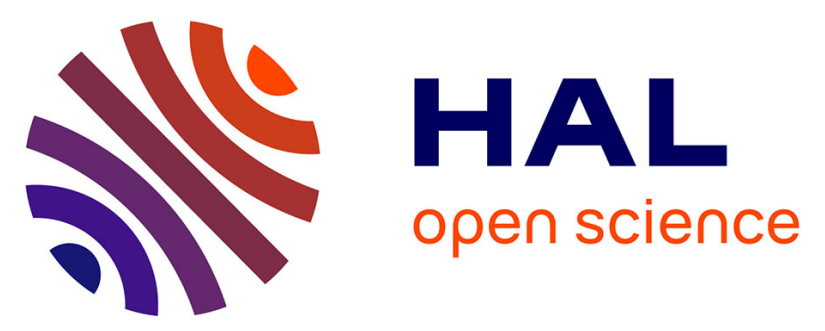

\title{
EFFICIENT MACHINE LEARNING FRAMEWORK FOR COMPUTER-AIDED DETECTION OF CEREBRAL MICROBLEEDS USING THE RADON TRANSFORM
}

Amir Fazlollahi, Fabrice Meriaudeau, Victor L Villemagne, Christopher Rowe, Paul Yates, Olivier Salvado, Pierrick T. Bourgeat

\section{To cite this version:}

Amir Fazlollahi, Fabrice Meriaudeau, Victor L Villemagne, Christopher Rowe, Paul Yates, et al.. EFFICIENT MACHINE LEARNING FRAMEWORK FOR COMPUTER-AIDED DETECTION OF CEREBRAL MICROBLEEDS USING THE RADON TRANSFORM. ISBI'2014, Apr 2014, Beijing,

China. pp.11"-116. hal-00989915

\section{HAL Id: hal-00989915 https://hal.science/hal-00989915}

Submitted on 12 May 2014

HAL is a multi-disciplinary open access archive for the deposit and dissemination of scientific research documents, whether they are published or not. The documents may come from teaching and research institutions in France or abroad, or from public or private research centers.
L'archive ouverte pluridisciplinaire HAL, est destinée au dépôt et à la diffusion de documents scientifiques de niveau recherche, publiés ou non, émanant des établissements d'enseignement et de recherche français ou étrangers, des laboratoires publics ou privés. 


\title{
EFFICIENT MACHINE LEARNING FRAMEWORK FOR COMPUTER-AIDED DETECTION OF CEREBRAL MICROBLEEDS USING THE RADON TRANSFORM
}

\author{
Amir Fazlollahi ${ }^{1,2}$, Fabrice Meriaudeau ${ }^{2}$, Victor L. Villemagne ${ }^{3}$, Christopher C. Rowe ${ }^{3}$, Paul Yates ${ }^{3}$, Olivier Salvado ${ }^{1}$, \\ Pierrick Bourgeat ${ }^{1}$ on behalf of the AIBL Research Group ${ }^{4}$ \\ ${ }^{1}$ CSIRO Preventative Health Flagship, CSIRO Computational Informatics, Herston, QLD, Australia, \\ ${ }^{2}$ Le2I, University of Burgundy, Le Creusot, France, \\ ${ }^{3}$ Department of Nuclear Medicine and Centre for PET, Austin Hospital, Melbourne, VIC, Australia, \\ ${ }^{4} \mathrm{http}: / / w w w . a i b l . c s i r o . a u /$
}

\begin{abstract}
Recent developments of susceptibility weighted MR techniques have improved visualization of venous vasculature and underlying pathologies such as cerebral microbleed (CMB). CMBs are small round hypointense lesions on MRI images that are emerging as a potential biomarker for cerebrovascular disease. CMB manual rating has limited reliability, is time-consuming and is prone to errors as small CMBs can be easily missed or mistaken for venous crosssections. This paper presents a computer-aided detection technique that utilizes a novel cascade of random forest classifiers which are trained on robust Radon-based features with an unbalanced sample distribution. The training samples and their associated bounding box were acquired from a multiscale Laplacian of Gaussian technique with respect to their geometric characteristics. Validation results demonstrate that the current approach outperforms state of the art approaches with sensitivity of $92.04 \%$ and an average false detection rate of 16.84 per subject.

Key words: Cerebral Microbleeds, Susceptibility Weighted Imaging, Radon transform, multi-scale Laplacian of Gaussian, sphere detection
\end{abstract}

\section{INTRODUCTION}

C CEREBRAL microbleeds (CMBs) are small deposits of hemosiderin, corresponding to prior microscopic hemorrhages [1]. CMBs are spherical, relatively small with diameter size of 2 to $10 \mathrm{~mm}$ [2], and hypointense on magnetic resonance images (MRI). CMBs with their anatomical prevalence are emerging as a new diagnostic marker of cognitive impairment and dementia [3], stroke and intracerebral haemorrhages (ICH) [4] and cerebral amyloid angiopathy (CAA) [5].

Manual localization is the gold standard technique to assess incidence of CMBs. However, visual screening is time-consuming, subjective and has low reproducibility between observers [6]. Moreover, it is prone to errors as small CMBs can be easily missed, or mistaken for vessel cross-sections, especially in MR Susceptibility Weighted Imaging (SWI) due to the high sensitivity of this sequence to magnetic susceptibility [7].

This study was co-sponsored by the Burgundy Regional Council.
Recently, there has been growing interest in ComputerAided assessment of CMBs in MR images. Seghier et al. [8] developed a unified probabilistic normalizationsegmentation framework to mark CMBs as an extra prior class. A set of morphological constraints were also imposed on the extra tissue prior followed by another iteration of the probabilistic segmentation to limit false detections. The method achieved CMB detection sensitivity of $50 \%$ while no results were reported on false detection rate. Barnes et al. [9] proposed a technique that first identifies hypointensities using local and global statistical thresholding algorithms. A supervised support vector machines classifier was then trained on a set of shape features to maximize the sensitivity. This method achieved sensitivity of $81.7 \%$ with 107 false CMBs per subject. Kuijf et al. [10] identified spherical objects using the radial symmetry transform (RST) applied on both echoes of dual echo $\mathrm{T} 2 * \mathrm{w}$. Further anatomical and statistical constraints were then introduced to improve the detection rate and obtained sensitivity of $71.2 \%$ producing 17.2 false detections per subject. Bian et al. [11] developed an algorithm based on 2D RST evaluated on patients suffering from brain tumors with CMBs due to external beam radiation therapy. A 3D region growing followed by geometric feature examination was used on top of RST to improve the detection rate. A sensitivity of $86.5 \%$ and an average 45 false CMBs per patients was achieved. In their method more than 10 constraints were empirically established using 5 independent patients. Most recently Kuijf et al. [12] evaluated their approach on a larger cohort size by incorporating two parameters on RST responses in order to adjust for a desired sensitivity. They obtained sensitivities of $65 \%, 78 \%$ and $84 \%$ with 20,49 and 96 average false detections per subject, respectively. Various characteristics of these studies are listed in Table 1.

This paper presents a flexible framework for computeraided detection of CMBs in SWI. This method utilizes a cascade of machine learning random forest (RF) classifiers that are trained on a set of robust Radon-based features. The training CMB samples are obtained from a multi-scale Laplacian of Gaussian (LoG) technique with respect to their geometric characteristics. The LoG approach significantly 


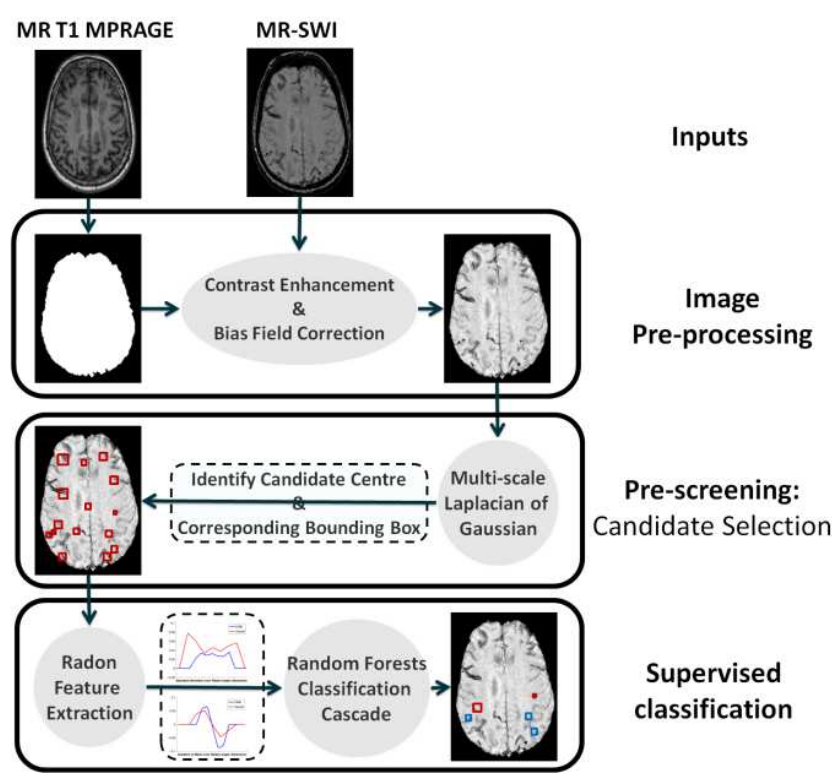

Fig. 1. Overview of the proposed processing pipeline

reduces the computational time required for analyzing $3 \mathrm{D}$ brain volume and also allows accurate bounding box estimation for the samples prior to feature extraction. (refer to Fig. 1)

Section II describes the dataset, candidate selection, feature extraction and classification pipeline. Section III presents results evaluated from sensitivity and false detection rates per subject. Finally, conclusion is described in Section IV.

\section{MATERIAL AND METHOD}

\section{A. Dataset}

This study included a data set of 7 subjects with Alzheimer's disease, 9 with mild cognitive impairment and 25 healthy controls from the Australian Imaging Biomarkers and Lifestyle study (AIBL) [1]. The subjects included 17 females (age 77.8 \pm 7.4 ) and 24 males (age 76.8 \pm 6.6 ).

For each subject, anatomical $\mathrm{T} 1 \mathrm{w}$ and SWI MR images were available. All MRIs were performed on a 3T Siemens TRIO scanner. 3D SWI was acquired with $0.93 \times 0.93 \mathrm{~mm}$ inplane resolution and $1.75 \mathrm{~mm}$ slice thickness, repetition time/echo time of $27 / 20 \mathrm{msec}$, and flip angle $20^{\circ}$. T1weighted images were acquired using a standard 3D magnetization-prepared rapid gradient echo sequence with in-plane resolution $1.0 \times 1.0 \mathrm{~mm}$, slice thickness $1.2 \mathrm{~mm}$, repetition-time/echo-time/T1 $=2.300 / 2.98 / 900$, flip angle $9^{\circ}$, field of view $240 \times 256$, and 160 slices.

The SWIs were manually labeled by two clinical experts using the Microbleed Anatomical Rating Scale (MARS) [6]. A consensus was then attained for presence and number of lesions. For $\mathrm{CMB}$ presence, the inter-observer agreement was $\kappa=0.65, \mathrm{p}<0.001$; reader 1 consensus $\kappa=0.8, \mathrm{p}<0.001$; reader 2 consensus $\kappa=0.83, \mathrm{p}<0.001$.

\section{B. Pre-processing}

The T1w image for each subject was first segmented into gray/white matter and CSF using the expectation maximization segmentation algorithm. The resulting segmentations were then combined to generate a skullstripping mask which was then rigidly aligned to the SWI image through block-matching. The SWI was also corrected for bias field using N4 [1]. To enhance the contrast and normalize the intensity across subjects, the dynamic intensity range of the skull-stripped SWI was normalized to $[0,1]$ after trimming the top $1 \%$ of intensity values. The image was then inverted so that CMBs and vessels appear hyperintense. To reduce noise and locally enhance the contrast, gradient based anisotropic diffusion and adaptive histogram equalization were applied on the SWIs.

\section{Candidate Pre-screening}

The analysis of image volumes to find all cubic regions-ofinterest (ROI) containing CMBs is a crucial step to spatially limit the high order analysis and computation time. We use a multi-scale LoG spherical/semi-spherical detection method with high sensitivity and low false detection rate [1]. The method has 3 steps:

- Breaking down 3D sphere detection into multi-scale 1D line detection along the $\mathrm{x}, \mathrm{y}$ and $\mathrm{z}$ dimensions.

- Identifying the center of any geometric shape by combining normalized line responses obtained in the previous step.

- Employing eigenvalues of the Hessian matrix of the extracted center points in order to determine spherical objects.

Validation results in [1] demonstrate that the current approach has higher performance in terms of sensitivity and specificity and is effective in detecting CMB candidates with invariance to intensity, orientation, translation and object scale. More importantly, it is capable of detecting CMBs adjacent to a neighboring vessel.

\section{Candidate Bounding Box}

To extract a set of shape features, a 3D bounding box needs to be defined per candidate proportional to its size. Radonbased features achieve a high response for spherical objects

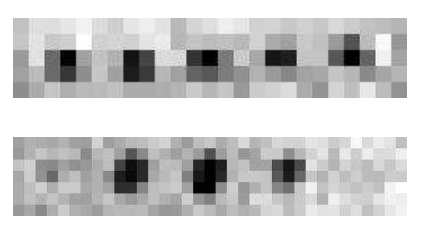

Fig. 2. The top left cell shows 5 slices of a 3D ROI of a vessel $(5 \times 5 \times 5)$ and $\mathrm{CMB}(7 \times 7 \times 5)$ selected in pre-screening phase. The image slices are placed side by side for viewing purposes. The right column illustrates the mean and standard deviation feature descriptor used by the classifier.
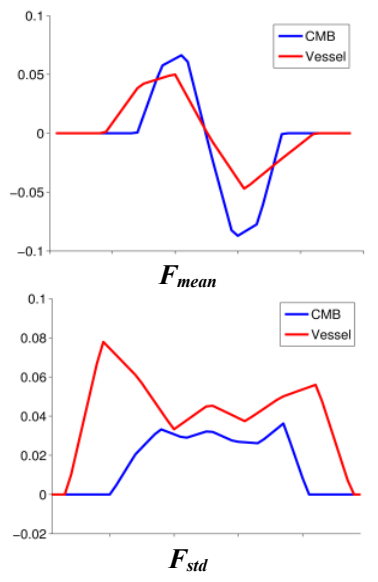


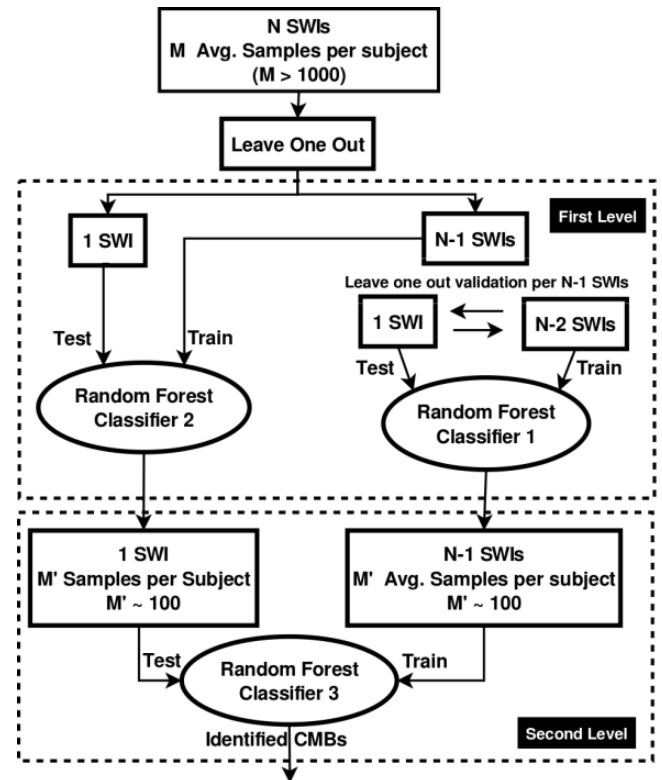

Fig. 3. Outline for a 2-layer classification cascade with leave-one-out scheme in subject level used in this paper.

once it is placed at the center of the ROI. The bounding box should be centered on the target, be small enough to enclose the entire extent of target structure while preserving sufficient background information.

In the current dataset with an abundant presence of small CMBs with in-plane size of $2 \times 2$ pixels, an ideal bounding box as characterized above could not be defined.

To address this issue, the SWI was up-sampled by a factor of 2 in every direction through linear interpolation. The multi-scale LoG is again performed locally on the upsampled image to estimate the size of the nominated candidate from the previous step. To achieve intensity shift invariance, the top and bottom $5 \%$ of the intensity values are removed and the result rescaled to $[0,1]$.

\section{E. Feature Extraction}

The Radon-based analysis has been shown to be effective in detecting Gaussian-like structures in the presence of noise or other structures in 2-dimensional images [1]. The Radon transform is capable of representing significant shape characteristics through an object's directional property. This transform can be written mathematically as mapping an image $f(x, y, z)$ to function $R f(\rho, \theta, \varphi)$. Let $\vec{n}=(\sin \theta \cos \varphi$, $\sin \theta \sin \varphi, \cos \varphi), \vec{p}=(x, y, z)$ a point on the image with image center as origin and $\vec{p} \cdot \vec{n}$ the equation of a plane for a given polar angle $\alpha=(\theta, \varphi)$, then the $3 \mathrm{D}$ radon transform of a image volume $f$ is defined as:

$$
R f(\rho, \alpha)=\sum_{-x}^{+x} \sum_{-y}^{+y} \sum_{-z}^{+z} f(x, y, z) \delta(\vec{p} \cdot \vec{n}-\rho)
$$

where $\delta$ is the Dirac function. This projection is able to highlight geometric shapes described by the parameters $(\rho, \alpha)$ where $\rho$ can be interpreted as the projection distance from the volume center.
Every image coordinate $(x, y, z)$ is mapped to physical space by defining the center of the image as origin and including image spacing. Keeping the generality of the Radon projection outputs, the results are organized in 2dimensional fashion with axes of $\rho$ and $\alpha$.

In [1] a set of Radon-based features was proposed to detect round structures in a sliding window in the presence of noise or other structures. These features are mean and standard deviation profile computed across the Radon angledimension:

$$
\begin{aligned}
& F_{\text {mean }}\left(x^{\prime}\right)=\frac{1}{M+N} \sum_{\alpha=1}^{M+N} R f\left(x^{\prime}, \alpha\right), \quad 1 \leq x^{\prime} \leq|\rho| \\
& F_{\text {std }}\left(x^{\prime}\right)=\sqrt{\frac{1}{M+N-1} \sum_{\alpha=1}^{M+N}\left(\frac{1}{|\rho|} \sum_{x=1}^{\rho} R f\left(x^{\prime}, \alpha\right)-F_{\text {mean }}\left(x^{\prime}\right)\right)^{2}}
\end{aligned}
$$

Where $|\rho|$ is the number of projection distances which is dependent on the ROI size, $M$ and $N$ are number of projection angles of polar coordinates $\theta$ and $\varphi$ where here are assigned to: $(\theta, \varphi) \in[0: 15: 165]$. (Fig. 2)

Feature descriptors extracted from the Radon should be scale, rotation and translation invariant. The proposed Radon descriptors are invariant to rotation by nature. Scale invariance is obtained by resizing $F_{\text {mean }}$ and $F_{\text {std }}$ to a fixed length. Translation invariance is enforced by placing the candidate CMBs at the center for the ROI prior to the Radon transform.

\section{F. Classification}

The capability of the proposed Radon features for identifying CMBs has been tested using binary RF classifier. High sensitivity of the candidate selection step to irregular and low contrast CMBs, adversely affects the training set to include several non-informative samples due to noise. This consequently results in an unbalanced training set with a class ratio greater than 277 .

The RF is able to handle large datasets with extremely unbalanced sample prevalence by incorporating class weights where misclassification of the minority class is penalized. However, the classification specificity is decreased due to the high presence of non-informative candidates in any randomly selected RF training set.

In the current work, a multi-layer classification cascade, composed of several RF classifiers, is proposed to

TABLE I

COMPARISON OF LITERATURE

\begin{tabular}{lcccccc}
\hline \hline Method & {$[\mathbf{8}]$} & {$[9]$} & {$[10]$} & {$[11]$} & {$[12]$} & $\begin{array}{c}\text { Proposed } \\
\text { method }\end{array}$ \\
Year & 2011 & 2011 & 2012 & 2013 & 2013 & $\mathbf{2 0 1 3}$ \\
MRI & $\mathrm{T} 2 *$ & $\mathrm{SWI}$ & $\mathrm{T} 2 *$ & $\mathrm{SWI}$ & $\mathrm{T} 2 *$ & SWI \\
modality & $1.5 \mathrm{~T}$ & $1.5 \mathrm{~T}$ & $7.0 \mathrm{~T}$ & $3.0 \mathrm{~T}$ & $3.0 \mathrm{~T}$ & $\mathbf{3 . 0 T}$ \\
Mag. Field & $\mathrm{Ctroke}$ & $\mathrm{AD} / \mathrm{MCI}$ & $\mathrm{CerV} 1$ & $\mathrm{Tumor}$ & $\mathrm{AD} / \mathrm{MCI}$ & $\mathbf{A D} / \mathbf{M C I}$ \\
Pathology & 30 & 6 & 18 & 10 & $38 / 72^{2}$ & $\mathbf{4 1}$ \\
\# Subjects & 114 & 126 & 66 & 304 & 148 & $\mathbf{1 0 3}$ \\
\# CMBs & $50 \%$ & $81.7 \%$ & $71.2 \%$ & 86.5 & $78 \%$ & $\mathbf{9 2 . 0 4 \%}$ \\
Sensitivity & - & 107 & 17.2 & 44.9 & 49 & $\mathbf{1 6 . 8 4}$ \\
Avg. FPs & - & & & & \\
\hline \hline
\end{tabular}

1) Cerebrovascular Disease

2) Number of pathological cases were 38 out of 72 patients where $71 \%$ of the CMBs were definite 


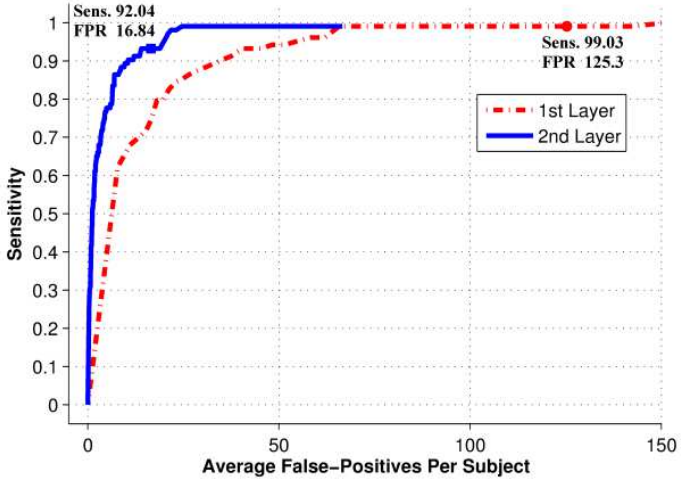

Fig. 4. The FROC curve for the full process comparing one and two layer cascade classifier.

progressively rule out non-informative samples and then train the final level on a relatively more balanced dataset. Binary RF posterior probabilities control classification performance and applying a low value in initial layers can reject obvious candidates. The leave-one-out validation scheme was extended for the classification pipeline to ensure that the subject's observation would not be part of the training sets. Fig. 3 shows the outline for a two-layer classification cascade approach.

\section{EXPERIMENTS AND RESULTS}

A total of 104 definite CMBs were identified from 41 SWIs with $\mathrm{CMB}$ prevalence of $2.36 \pm 3.09$ per subject. One CMB was excluded due to its irregular shape (Fig. 5.a).

In the pre-screening step, in order to achieve sufficiently high sensitivity and specificity for candidates, the intensity and sphericalness parameters were empirically explored and set to 0.55 . The multi-scale LoG smoothing kernels were assigned to [0.8:5.0] with a step size of 0.3 to comply with CMB diameter size of 2 to $10 \mathrm{~mm}$. For the classification step, 2 cascades of RF classifiers were employed with leaveone subject-out validation scheme. For the first classifier, the threshold on the posterior probability was chosen as 0.1 to ensure removing a sufficient number of CMB mimics while preserving most of the true CMBs.

The pre-screening step has a very high sensitivity of $\sim 98 \%$, leaving 2 undetected CMBs out of 103 and produces on average 695 candidates per subject. Fig. 4 illustrates the FROC curve obtained from 1- and 2-layer classifiers. The best performance for the full process was achieved by 2 layer cascade with an overall sensitivity of $92.04 \%$
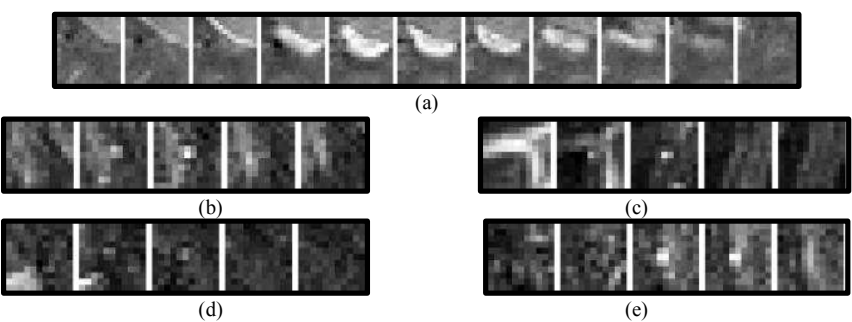

Fig. 5. Slices of $3 \mathrm{D}$ ROIs containing CMBs are placed side by side for visualization. (a) Shows a CMB that was excluded due to irregular shape. (b)-(e) are examples of false negatives resulting from the proposed method. producing on average 6.7 false-positives per true CMB and 16.84 false CMBs per subject. Fig. 5 shows few false negative examples resulting from the proposed method. The quantitative results of the proposed technique along with similar in the literature are summarized in Table 1. However, performance of published studies depends on the dataset and can be biased due to imaging parameters, CMB appearance, underlying disease and the manual identifications.

\section{CONCLUSION}

This paper presented a flexible framework to automatically detect cerebral microbleeds on MR images with a high sensitivity and a low false detection rate. This method can therefore be used to speed up manual identification of CMBs. Furthermore, it improves reliability and minimizes the inter-rater variability that is widely reported in manual ratings. As future work, the proposed method can be further improved by including more CMBs in the training set and incorporating Hessian shape descriptors in the feature set.

\section{REFERENCES}

[1] S. Greenberg, M. Vernooij, C. Cordonnier et al., "Cerebral microbleeds: a guide to detection and interpretation," The Lancet Neurology, vol. 8, no. 2, pp. 165-174, 2009.

[2] C. Cordonnier, R. Salman, and J. Wardlaw, "Spontaneous brain microbleeds: systematic review, subgroup analyses and standards for study design and reporting," Brain, vol. 130, no. 8, pp. 1988-2003, 2007.

[3] P. Yates, R. Sirisriro, V. Villemagne, S. Farquharson, C. Masters, C. Rowe et al., "Cerebral microhemorrhage and brain $\beta$-amyloid in aging and Alzheimer disease," Neurology, vol. 77, no. 1, pp. 48-54, 2011.

[4] J. Fiehler, "Cerebral microbleeds: old leaks and new haemorrhages," International Journal of Stroke, vol. 1, no. 3, pp. 122-130, 2006.

[5] Y. Nakata-Kudo, T. Mizuno, K. Yamada et al. "Microbleeds in Alzheimer disease are more related to cerebral amyloid angiopathy than cerebrovascular disease," Dementia and geriatric cognitive disorders, vol. 22, no. 1, pp. 8-14, 2006.

[6] S. Gregoire, U. Chaudhary, M. Brown, T. Yousry, C. Kallis, H. Jäger, and D. Werring, "The Microbleed Anatomical Rating Scale (MARS)," Neurology, vol. 73, no. 21, pp. 1759-1766, 2009.

[7] M. Ayaz, A. S. Boikov, E. M. Haacke, D. K. Kido, and W. M. Kirsch, "Imaging cerebral microbleeds using susceptibility weighted imaging: one step toward detecting vascular dementia," Journal of Magnetic Resonance Imaging, vol. 31, no. 1, pp. 142$148,2010$.

[8] M. Seghier, M. Kolanko, A. Leff et al. , "Microbleed detection using automated segmentation (MIDAS): a new method applicable to standard clinical MR images," PloS one, vol. 6, no. 3, p. e17547, 2011.

[9] S. Barnes, E. Haacke, M. Ayaz, A. Boikov, W. Kirsch, and D. Kido, "Semiautomated detection of cerebral microbleeds in magnetic resonance images," Magnetic Resonance Imaging, 2011.

[10] H. J. Kuijf, J. de Bresser, M. I. Geerlings, et al., "Efficient detection of cerebral microbleeds on 7.0 T MR images using the radial symmetry transform," Neuroimage, vol. 59, no. 3, pp. 2266-2273, 2012.

[11] W. Bian, C. P. Hess, S. M. Chang, S. J. Nelson, and J. M. Lupo, "ComputerAided Detection of Radiation-Induced Cerebral Microbleeds on SusceptibilityWeighted MR Images," NeuroImage: Clinical, 2013.

[12] H. J. Kuijf, M. Brundel, J. de Bresser, et al., "Semi-automated detection of cerebral microbleeds on 3.0 T MR images," PLoS ONE, vol. 8, no. 6, p. e66610, 2013. [13] K. A. Ellis, A. I. Bush, D. Darby et al., "The Australian Imaging, Biomarkers and Lifestyle (AIBL) study of aging: methodology and baseline characteristics of 1112 individuals recruited for a longitudinal study of Alzheimer's disease," International Psychogeriatrics, vol. 21, no. 04, pp. 672-687, 2009.

[14] N. Tustison, B. Avants, P. Cook, Y. Zheng et al., and J. Gee, "N4itk: improved N3 bias correction," Medical Imaging, IEEE Transactions on, vol. 29, no. 6, pp. 1310 1320, 2010.

[15] A. Fazlollahi, F. Meriaudeau, V. Villemagne et al., "Automatic detection of small spherical lesions using multiscale approach in 3D medical images," in International Conference on Image Processing (ICIP). IEEE, 2013, pp. 1158-1162.

[16] L. Giancardo, F. Meriaudeau, T. Karnowski et al., "Microaneurysm detection with radon transform-based classification on retina images," in Engineering in Medicine and Biology Society, EMBC, 2011 Annual International Conference of the IEEE. IEEE, 2011, pp. 5939-5942. 